\section{El problema del enfoque médico de la discapacidad: Un desafío interdisciplinario entre salud, educación y el derecho}

\section{The problem of medical approach to disability: An interdisciplinary challenge between health, education and law}

\section{Sr. Editor:}

Los distintos enfoques con que puede abordarse la discapacidad, y el impacto social que estos conllevan, demanda la necesidad que disciplinas tan distintas como salud, justicia y educación, trabajen de manera interdisciplinaria para definir una relación entre sujeto y sociedad basada en el respeto y el derecho. La relevancia que tiene la salud (física y mental) para la participación social, permite inquirir respecto de cómo sus enfoques influyen en el ejercicio de los derechos de las personas con discapacidad.

En este sentido, los derechos humanos han debido clasificarse en tres generaciones ${ }^{1}$. Una primera generación, constituida por aquellos orientados a garantizar libertades individuales como el derecho a la vida y la identidad. Una segunda generación, constituida por derechos económicos, sociales y culturales, entre los que se encuentran los derechos a salud y educación; y finalmente, la tercera y más actual generación, que responde a tensiones producidas por aspectos relacionales y contextuales. Los derechos a la paz, del consumidor, a la calidad de vida y la libertad informática, se constituyen dentro de este grupo.

Estos derechos de tercera generación, están orientados a responder a relaciones de poder que a su vez promueven una ideología de mundo homogéneo ${ }^{2}$. En este contexto, el fenómeno de la discapacidad se ha constituido exclusivamente desde enfoques médicos los cuales han sido tensionado con los enfoques de derecho ${ }^{3}$. La distinción fundamental se encuentra en que mientras el primer enfoque comprende la discapacidad como una afección al natural desempeño de la persona, el enfoque de derecho la comprende desde la interacción con las barreras y oportunidades sociales.

Si bien se han hecho esfuerzos desde los organismos de salud por superar este enfoque, las definiciones más recientes siguen perpetuando lo negativo de la discapacidad, englobando en ella, deficiencias, limitaciones y restricciones para la participación y la actividad ${ }^{4}$. Situar por el contrario, la discapacidad desde un enfoque de derecho, contribuiría a asumir que los contextos disponen de barreras para la participación, disminuyendo la connotación negativa que históricamente ha asimilado la discapacidad a una enfermedad diagnosticable en el sujeto.
Las consecuencias del enfoque médico, derivan en una concepción social que entiende la discapacidad con temor, lástima e inseguridad. Esto se ha declarado en el informe emitido por el Comité sobre los Derechos de las Personas con Discapacidad ${ }^{5}$ el cual establece su preocupación por la persistencia del modelo médico en instrumentos chilenos como el Código Civil, la política de salud mental, así como los modelos asistencialistas reforzados por campañas como Teletón.

Estas concepciones, se ven luego reflejadas en la educación, la cual ha heredado en su política el enfoque médico, entendiendo que la discapacidad debe ser diagnosticada y mejorada para que el sujeto normalizado pueda adaptarse al sistema ${ }^{6}$. La preocupación por reproducir sin mayor reflexión el enfoque médico de la discapacidad, radica en que la educación conduce hacia otras formas de participación ciudadana como son el trabajo, la familia y la vida en comunidad.

El poder que las ciencias de la salud, jurídicas y de la educación, ejercen en el discurso y las representaciones sociales sobre la discapacidad,sitúa el desafío de articular los saberes de estas tres disciplinas, en vías de un concepto de discapacidad que pueda transitar desde un foco centrado en las limitaciones del sujeto, hacia una valoración del mismo en tanto sujeto diferente que interactúa con una sociedad que no ha sido diseñada para convivir con la diferencia.

El derecho a la calidad de vida, en el cual la persona con discapacidad se reconoce a sí misma y es reconocida por otros, como sujeto particular y no como alteración a la norma, permitiría construir una sociedad en la cual el ejercicio de los derechos a la educación y salud se garantice desde la diferencia, entendiendo al sujeto conforme a sus múltiples características, cosmovisiones y formas de ser y conocer en el mundo. El trabajo interdisciplinario entre las ciencias de la salud, jurídicas y de educación, en tanto promotoras del bienestar social, es un aspecto fundamental para avanzar en una sociedad cada vez más inclusiva.

\section{Referencias bibliográficas}

1. Pérez AE. Las generaciones de derechos humanos. Centro de Estudios Constitucionales 1991; 10 (1): 203-19.

2. Freedman R. Third Generation's Rights: Is there room for hybrid constructs within international human right law? Cambridge Journal of International and Comparative Law 2013; 2 (4): 935-59.

3. Hernández MI. El concepto de discapacidad: De la enfermedad al enfoque de derechos. Revista CES Derecho 2015; 6 (2): 46-59.

4. OMS (2011). Resumen. Informe mundial sobre la discapacidad. Disponible: http://www.who.int/disabilities/world_report/2011/accessible_es.pdf [Consultado el 24 de octubre de 2016]. 
5. ONU (2016). Comité sobre los derechos de las Personas con Discapacidad. Observaciones finales sobre el informe inicial de Chile. Disponible: http://acnudh.org/wp-content/ uploads/2016/04/CRPD_C_CHL_CO_1_23679_S.pdf [Consultado el 24 de octubre de 2016].

6. Peña M. Análisis crítico del discurso del Decreto 170 de Subvención Diferenciada para Necesidades Educativas Especiales: El diagnóstico como herramienta de gestión. Psicoperspectivas, Individuo y Sociedad 2013; 12 (2): 93-103.

\section{Jorge Eduardo Alfaro Urrutia Profesor en Educación Diferencial, Magíster en Educación, Estudiante del Programa de Doctorado en Ciencias de la Educación de la Universidad de la Frontera, Temuco Chile.}

El manuscrito presentado forma parte de la problematización de la tesis doctoral del autor respecto de inclusión escolar.

Fuente de apoyo financiero: Comisión Nacional de Investigación Científica y Tecnológica CONICYT en el marco de la Beca de Doctorado Nacional año 2015. La Comisión no tuvo influencia en el diseño, recolección, análisis o interpretación de datos, ni en la elaboración del manuscrito.

Correspondencia a:

Jorge Eduardo Alfaro Urrutia

Educador Diferencial

Universidad de la Frontera, Temuco Chile

jorgeeduardoau@gmail.com

\section{Retroalimentación: Piedra angular de la enseñanza clínica}

\section{Feedback: Cornerstone of clinical teaching}

\section{Sr. Editor:}

En la educación médica es fundamental la práctica clínica supervisada con pacientes, bajo la supervisión de tutores clínicos, y en la cual la retroalimentación brindada por ellos es la piedra angular de una enseñanza efectiva ${ }^{1}$.

Es claro que sin una retroalimentación adecuada en tal práctica, los desempeños sobresalientes no son reforzados, y podrían perpetuarse desempeños deficientes ${ }^{2}$, afectando en último término la atención de los pacientes; hay evidencias de que este proceso no llevado a cabo efectivamente y los estudiantes refieren no recibir suficiente retroalimentación ${ }^{3}$.

Dentro de las condiciones que dificultan este proceso, se menciona que los clínicos deben atender múltiples tareas que compiten con la enseñanza clínica, como la atención de pacientes, investigar, publicar, realizar tareas de extensión y dedicar tiempo a su perfeccionamiento continuo ${ }^{4}$. También se han descrito dificultades en una inadecuada recepción de la retroalimentación por parte de los estudiantes, que pueden responder en forma defensiva a una determinada observación de su desempeño por una inadecuada capacidad metacognitiva, descrita como pensar sobre los propios pensamientos y sentimientos ${ }^{3}$.

Entonces, considerando la importancia de una buena retroalimentación en la educación médica, las escuelas de medicina deberían formar a sus tutores clínicos entregándoles una preparación académica formal, obligatoria y basada en la evidencia existente, además del reconocimiento y los estímulos adecuados para que puedan ejercer esta importante función de la mejor forma y mejorar así el actual nivel de docencia en nuestras escuelas.

Nicolás Ortiz-López Estudiante de Medicina, Facultad de Medicina, Universidad de Chile, Santiago, Chile. Subdirector de Investigación, Programa de Ayudantes Docentes, Facultad de Medicina, Universidad de Chile, Santiago, Chile.

\section{Referencias}

1. Cantillon P, Sargeant J. Giving feedback in clinical settings. BMJ 2008; 337: 1292-4.

2. Ceccarelli JF. Feedback en educación clínica. Rev Estomatol Hered 2014; 24 (2): 127-32.

3. Bing-You RG, Trowbridge RL. Why Medical Educators May Be Failing at Feedback? JAMA 2015; 302 (12): 22-3.

4. Herskovic P. La formación pedagógica de académicos clínicos en la Facultad de Medicina de la Universidad de Chile. Rev Hosp Clínico Univ Chile 2009; 20: 315-8.

Declaro no tener conflictos de intereses.

Correspondencia a:

Nicolás Ortiz López

Gamero 1421, Departamento 1606, Independencia, Santiago,

Chile.

nicolas.ortiz@ug.uchile.cl 